\title{
Composites with Inclusions of Negative Bulk Modulus: Extreme Damping and Negative Poisson's Ratio
}

\author{
Y. C. WANG ${ }^{1}$ AND R. S. LAKES ${ }^{2}$ * \\ ${ }^{1}$ MST-8, MS G755, Los Alamos National Laboratory, Los Alamos, NM 87545, USA \\ ${ }^{2}$ Department of Engineering Physics, Engineering Mechanics Program \\ Biomedical Engineering Department, Materials Science Program and Rheology \\ Research Center, University of Wisconsin-Madison \\ 147 Engineering Research Building \\ 1500 Engineering Drive, Madison, WI 53706-1609, USA
}

(Received June 21, 2004)

(Accepted November 8, 2004)

\begin{abstract}
The effect of a negative bulk modulus phase in elastic composites is studied. Negative bulk modulus $K<0$ is shown to be possible in selected unit cells. In isotropic solids, $K<0$ can be attained when negative Poisson's ratio $v$ is sufficiently small, below the stability limit (for stress control) $v=-1$. Such materials, if used as inclusions, are predicted to be stable with respect to the band formation, even if they are large. Composites with spherical inclusions of negative bulk moduli are shown to exhibit negative Poisson's ratio and anomalies in composite bulk modulus and Young's modulus (and in the corresponding mechanical damping) but not in the shear modulus.
\end{abstract}

KEY WORDS: stability, viscoelasticity, negative Poisson's ratio, negative modulus.

\section{INTRODUCTION}

$\mathbf{F}$ OR MOST ELASTIC systems, stiffness is positive i.e., a deformed object experiences a force in the same direction as the deformation. Negative stiffness is possible in the systems, such as prestrained objects including postbuckled elements, which contain stored energy [1]. Heterogeneous systems with one constituent of negative stiffness are of interest since they are predicted to give rise to extreme overall damping and stiffness [2], high viscoelastic damping, and negative axial stiffness was observed [3] in compliant systems containing postbuckled tubes. High viscoelastic damping has also been observed in metal matrix $(\mathrm{Sn})$ composites containing $\mathrm{VO}_{2}$ particulate inclusions [4] which undergo a ferroelastic phase transformation. Ferroelastics are of interest in this context since they

*Author to whom correspondence should be addressed. E-mail: lakes@engr.wisc.edu 
are predicted to exhibit negative stiffness below the transformation temperature. Ferroelastics undergo a shear instability in which the crystal structure changes form as the temperature is lowered. This has been understood in the context of the free energy in the Landau theory having a relative maximum, corresponding to unstable equilibrium, below the material's transformation temperature $T_{c}$ [5]. Since the force is proportional to the gradient of the energy, the region near a relative maximum of energy corresponds to negative stiffness which is ordinarily not observed since shear instability in ferroelastics is associated with the formation of bands or domains [6] below a transition temperature $T_{c}$. Multidomain blocks of material are known to have positive stiffness, and are therefore not of interest in the context of inclusions in negative stiffness-based composites. To achieve negative shear modulus $G$ in a ferroelastic, the inclusion must be a single domain. Small particles may be single domains as a consequence of the surface energy penalty of the band formation. For some materials, the domain size can range from tens of $\mu \mathrm{m}$ up to several millimeters in size, but for other materials of interest, including shape memory alloys, the domains are tens of nanometer in size. Making and embedding granules of this size can be a challenge to the experimentalist, therefore, variants of negative stiffness not subject to banding instability are of interest.

The rationale for considering negative bulk modulus $K$ is that we can have $K<0$, and still have stability with respect to formation of bands by ensuring that $G>0$. Such a material is unstable with respect to global deformation. It can be stabilized by a constraint on its boundaries, in contrast to $G<0$ material. In a material with $K<0$ used in a composite, the constraint is supplied by the surrounding matrix.

\section{STABILITY OF A CONTINUUM: EFFECT OF BULK MODULUS}

In isotropic elastic solids, the 'allowable' range of Poisson's ratio $v$,

$$
-1<v<0.5
$$

corresponds to the requirement that the shear $(G)$ and bulk $(K)$ moduli [7] be positive for stability of an unconstrained block of material. The lack of constraint is equivalent to a surface traction boundary condition in the language of elasticity theory. Common materials have Poisson's ratios close to 0.3 , and rubbery materials have Poisson's ratios close to 0.5 . In view of the possibility of negative Poisson's ratio, foams [8,9] with $v$ as small as -0.8 have been conceptualized, fabricated, and studied. Negative Poisson's ratio differs from negative stiffness. Poisson's ratio is defined as the negative lateral strain of a stretched or compressed body divided by its longitudinal strain. Most materials when axially stretched will contract laterally, hence they have a positive Poisson's ratio. Poisson's ratio is dimensionless, and for most solids its value ranges between 0.25 and 0.33. Strictly, negative Poisson's ratio in a heterogeneous solid does not require an empty space, but a large contrast between constituent properties is required in a hierarchical composite realization [10]. As Poisson's ratio approaches -1 , the bulk modulus becomes much less than the shear modulus.

Strong ellipticity entails, $\boldsymbol{C}_{i j k l} \mathbf{n}_{i} \mathbf{n}_{k} \mathbf{m}_{j} \mathbf{m}_{l}>0$ for all nonzero vectors $\mathbf{n}$ and $\mathbf{m}$ and $\boldsymbol{C}_{i j k l}$ as the modulus tensor. The corresponding range of isotropic elastic constants, is [11]

$$
G>0 \quad \text { and } \quad v<0.5 \text { or } v>1 \text {. }
$$


Since, $E=2 G(1+v)$, this allows negative Young's modulus $E$ and bulk modulus $K$, specifically

$$
-\infty<E<\infty \quad \text { or } \quad-\frac{4 G}{3}<K<\infty
$$

The second condition ( 2 b) for strong ellipticity entails the constrained tensorial modulus be positive, $\boldsymbol{C}_{1111}>0$. Strongly elliptic materials are stable with respect to formation of bands.

Displacement-type boundary value problems have unique solutions if [12] the elastic constants are in the range for strong ellipticity. This range is considerably less restrictive than that for the traction condition. This means that a block of material constrained at the surface can have a negative bulk modulus or Young's modulus and be stable with respect to both global deformation and band formation.

We can have $K<0$ within strong ellipticity if, $\mu=G$ is sufficiently larger than $C_{1111}$ since [13].

$$
K=\lambda+\frac{2}{3} \mu=\lambda+\frac{6}{3} \mu-\frac{4}{3} \mu=\lambda+2 \mu-\frac{4}{3} \mu=C_{1111}-\frac{4}{3} \mu
$$

with $\lambda$ and $\mu$ as the Lamé constants.

The range of elastic moduli corresponding to stability and instability under various conditions is shown in the map in Figure 1. The representation of the upper right quadrant, as well as the term 'dilational' is due to Milton [10]. The stippled region corresponds to $-\infty<v<-1$ hence to $E<0$ as well as $K<0$. The shaded region corresponds to $G<0$ or $C_{1111}<0$ hence, a failure of strong ellipticity.

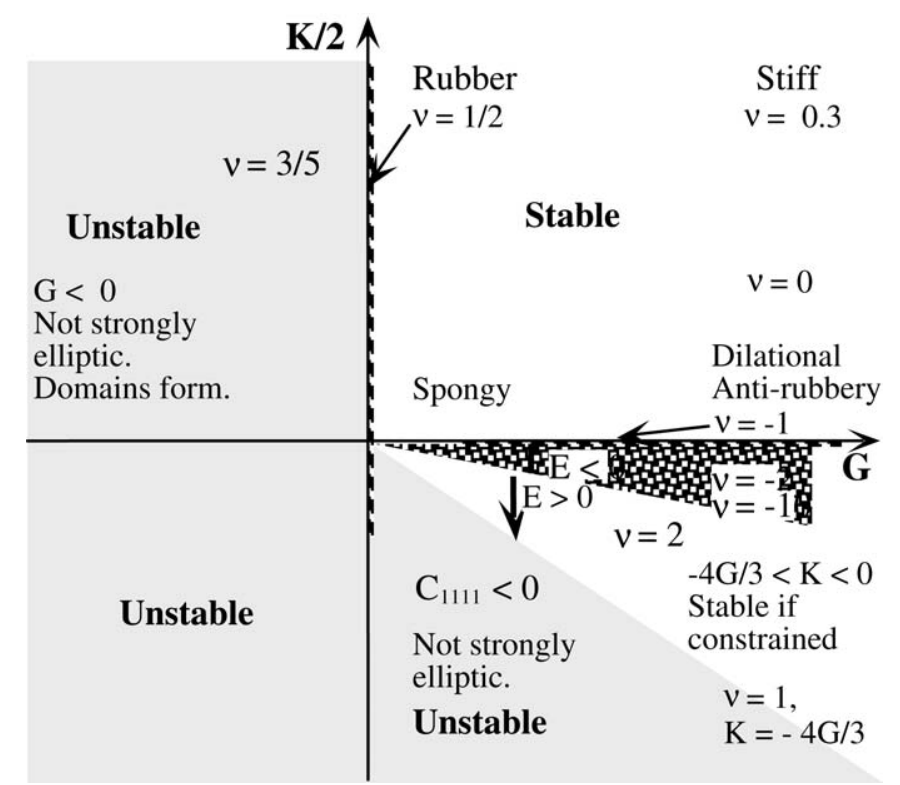

Figure 1. Map of elastic material properties corresponding to different values of bulk modulus $K$ and shear modulus $G$, allowing negative values. 


\section{NEGATIVE MODULI}

\section{Negative Spring Constant}

Negative stiffness is known to occur in the context of postbuckled structures and objects [14]. Stiffness of a lumped system refers to the ratio of force to deflection. A column which has been buckled into an ' $S$ '-shaped configuration is unstable and tends to snap through. By pressing laterally on the column, one can cause it to snap through. If the loaddeformation characteristic is studied in the displacement control (which entails a hard constraint), negative stiffness is observed experimentally. Negative stiffness also occurs in single-cell models of foam materials. Such models were observed experimentally to exhibit a compressive force-deformation relation which is not monotonic [15]. Inward bulge of cell ribs gives rise to a geometric nonlinearity.

\section{Negative Shear Modulus}

Prestrained lattice cells theoretically can give rise to negative Poisson's ratio and even negative shear modulus [16]. Such lattice structures were originally examined in a study of generalized continuum mechanics [17]. Negative shear modulus is also inferred from the behavior of ferroelastic and ferroelectric materials in the vicinity of phase transitions. As temperature is lowered to the transformation temperature $T_{c}$, the shear modulus softens to zero (or as close to zero as the experiment can resolve). Below $T_{c}$, bands or domains form in the material, as anticipated by the continuum theory and illustrated in the map in Figure 1. Single domain crystals are possible, based on a competition between the surface energy and the energy associated with domain boundaries.

\section{Negative Bulk Modulus}

\section{EXISTENCE}

Since negative bulk modulus materials are not subject to instability associated with domain formation, it is natural to ask whether such materials exist.

In crystalline materials, softening of the bulk modulus (analogous to softening of the shear modulus in ferroelastics) has been observed in $\mathrm{YbInCu}_{4}$ [18] crystals at a temperature of $67 \mathrm{~K}$. Cerium [19] exhibits a volumetric phase transformation [20] in response to low temperature (116 K at $1 \mathrm{~atm})$ or high pressure $(7670 \mathrm{~atm}$ at $298 \mathrm{~K})$. There is a $15 \%$ volume change with no change in crystal structure. Such transitions have been interpreted in the context of negative compressibility [21] hence, negative bulk modulus $K<0$. Consider in this vein the temperature-pressure phase diagram of tin [22]. Tin undergoes a transformation from tetragonal (white or $\beta$-Sn) to diamond structure (gray or $\alpha$-Sn) with a $26 \%$ volume increase. The transformation under temperature control is very slow [23]. Hydrostatic compression favors the high density white phase. Therefore, compression of gray tin under stress control will cause a snap through effect. In principle, if this compression were done under displacement control, a negative bulk modulus would occur. In general, a transformation temperature which decreases with increasing pressure will favor a snap through effect, provided the low temperature phase is less dense than the high density phase. Also, analysis [24] of an Ising model of a lattice predicts $K<0$ near the critical temperature. 
To illustrate effects which can occur in crystals, we consider a lattice (Figure 2), due to Berglund [17] who considered positive moduli in the context of generalized continuum mechanics. The lattice model allows for noncentral forces via crossed elastic bands which pass over the edges of circular nodes. The length $l_{0}$ of a band is given by $l_{0}=\sqrt{d^{2}-4 r^{2}}$, where $d$ is the spacing between node centers at equilibrium, and $r$ is the radius of a node. The spring constant of a vertical or horizontal spring is $k_{1}, k_{2}$ is that of a diagonal spring, and $k_{3}$ is that of a band. Prestrain in the lattice is provided as follows. The natural lengths of the elastic elements are $h_{1}, h_{2}$, and $h_{3}$, related to the lattice spacing $d$ by $h_{1}=f d$, $h_{2}=\sqrt{2} g h_{1}$. Prestrain is present if either $f$ or $g$ differs from 1 . Equilibrium of the nodes implies the remaining natural length is $h_{3}=\left(d / l_{0}\right)\left(1 / k_{3}\right)\left[\left(k_{2} / \sqrt{2}\right)\left(h_{2}-d \sqrt{2}\right)+\right.$ $\left.k_{1}\left(h_{1}-d\right)\right]+l_{0}$.

The structure is cubic, but elastic isotropy may be achieved by the following choice of the stiffnesses $k_{1}, k_{2}$, and $k_{3}$ of the elastic ligaments.

$$
k_{2}=\frac{h_{1} k_{1}}{\sqrt{2} h_{2}}+\frac{\sqrt{2} h_{3}\left(l_{0}^{2}-4 r^{2}\right)}{h_{2} l_{0} d} k_{3} .
$$

The governing equations for effective elastic properties are as follows:

$$
G=k_{1}+\left(2-\frac{3 h_{2}}{\sqrt{2} d}\right) k_{2}+\left(2-\frac{8 r^{2} h_{3}}{d^{2} l_{0}}\right) k_{3},
$$

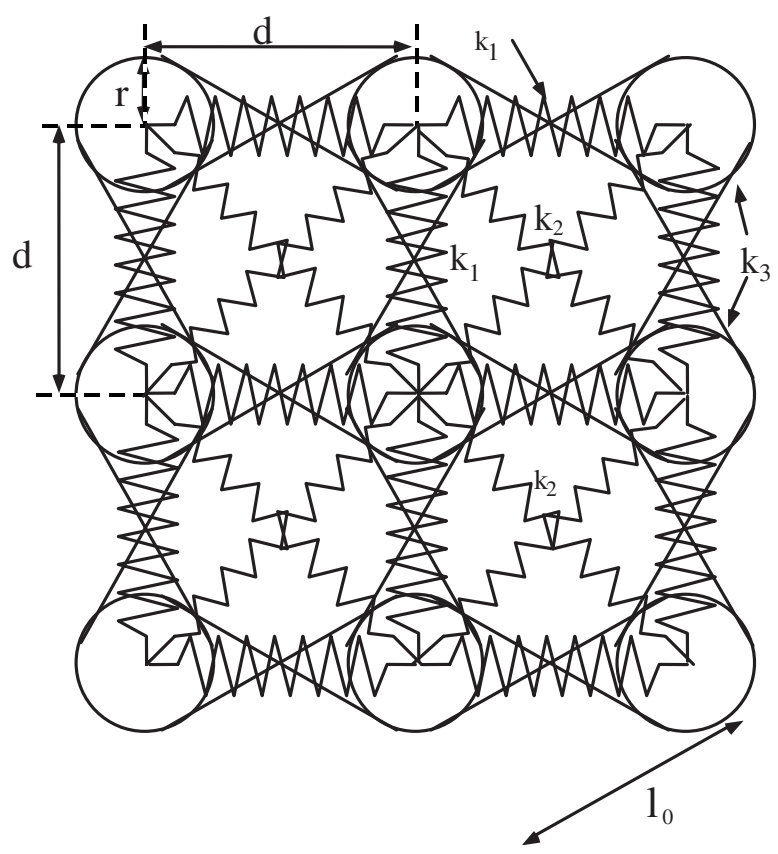

Figure 2. Lattice cell model. 


$$
\lambda=\sqrt{2} \frac{k_{2} h_{2}}{d}-G+2 \alpha,
$$

with $a=4 k_{3} r^{2} / d^{2}$. The bulk modulus $K$ is calculated via Equation (4). Poisson's ratio is given by

$$
v=\frac{3 K-2 G}{2(3 K+G)}, \quad \text { or } \quad v=\frac{\lambda}{2(\lambda+G)} .
$$

Here, $G, K$, and $v$ represent shear, bulk modulus, and Poisson's ratio, respectively. The lattice gives rise to a Poisson's ratio of $1 / 4$ if the circular nodes have zero size, giving rise to purely central forces provided there is no prestrain and the elastic isotropy condition (5) is satisfied. Introduction of prestrain in the bands (drawn as straight lines rather than as springs) can raise or lower the Poisson's ratio and can give rise to negative Poisson's ratio [25].

Results for an assumption of a lattice with elastic isotropy (Equation (5)) and $k_{1}=1$, $k_{3}=1, f=2 /((3-1) / g)$, and $r / d=0.1$ are as shown, as a function of prestrain ratio, in Figure 3. In this example, preload in $k_{3}$ is not needed, so $f$ is related to $g$ to balance the preload in $k_{1}$ and $k_{2}$. As shown in Figure 3, if the prestrain is of sufficient magnitude, moduli can become negative. Small $g$ corresponds to the stretching of the diagonal $k_{2}$ elements; for sufficiently small $g$, the shear modulus $G$ becomes negative and Poisson's ratio exceeds $1 / 2$. Large $g$ corresponds to the compression of these elements; for

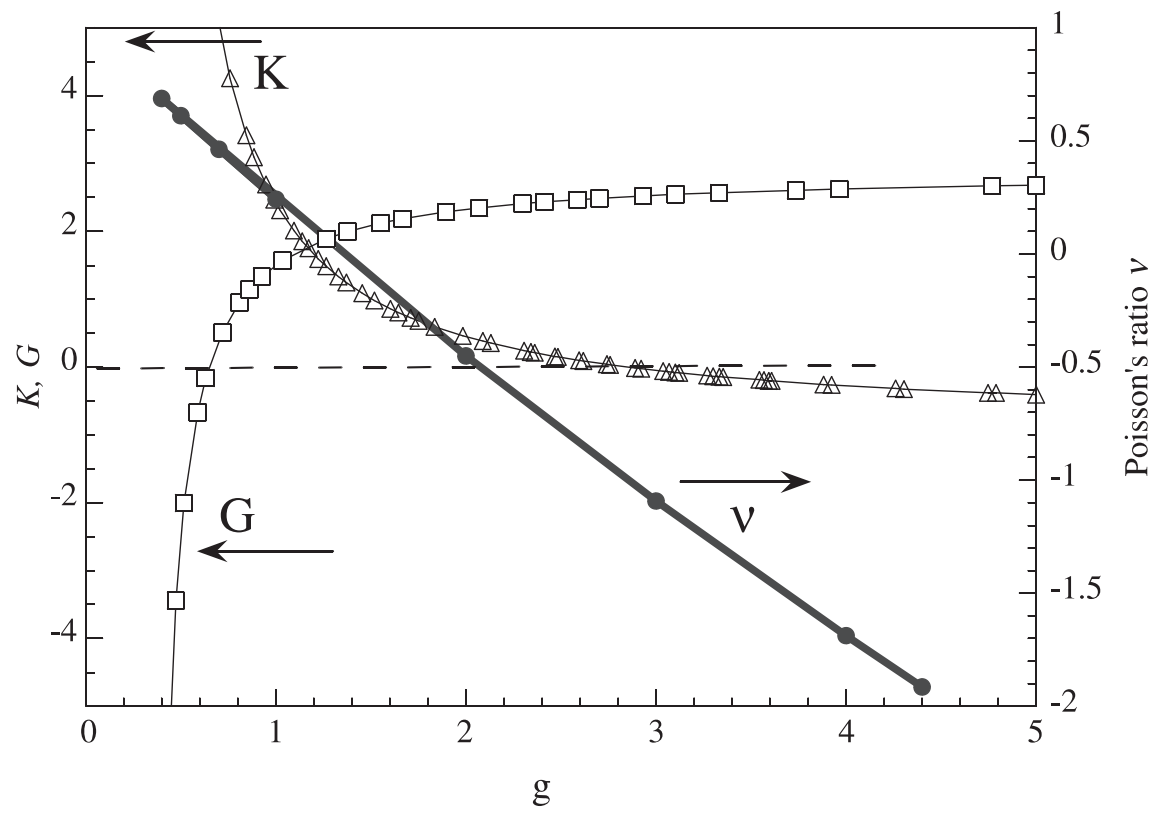

Figure 3. Elastic properties of a lattice model as a function of prestrain in the diagonal elements as specified by dimensionless ratio $\mathrm{g} ; \mathrm{g}=1$ corresponds to no prestrain. Shear modulus $\mathrm{G}$ (squares) and bulk modulus $\mathrm{K}$ (triangles) are normalized to $k_{1}$. Dash line indicates zero modulus. Poisson's ratio v (solid circles) can be negative and can exceed the stability limits for a stress boundary condition. 
sufficiently large $g$, the bulk modulus $K$ becomes negative and Poisson's ratio is less than -1 . The diagonal stiffness $k_{2}$, determined by the isotropy condition (5), remains positive throughout the range of $g$ in Figure 3.

In summary, negative bulk modulus may be inferred from the behavior of certain materials in the vicinity of phase transformations as well as from a lattice model of a crystal.

\section{STABILITY}

While it is known that an object with negative bulk modulus can be stable if constrained on all its boundaries, it is of interest in the context of potential experiments whether it might be stable under partial constraint as in a tension/compression test. We therefore consider stability of negative bulk modulus in the context of a body under displacement constraint along two parallel faces, as is done in a measurement of Young's modulus in displacement control. In this case, the lateral surfaces are under a specified stress of zero. Several cases of transverse deformation are considered in the context of stability under controlled transverse stress.

Consider the elementary isotropic form for Hooke's law with $\varepsilon$ as strain and $\sigma$ as stress.

$$
\begin{aligned}
& \varepsilon_{X X}=\left(\frac{1}{E}\right)\left\{\sigma_{X X}-v \sigma_{Y Y}-v \sigma_{Z Z}\right\} \\
& \varepsilon_{Y Y}=\left(\frac{1}{E}\right)\left\{\sigma_{Y Y}-v \sigma_{X X}-v \sigma_{Z Z}\right\} \\
& \varepsilon_{Z Z}=\left(\frac{1}{E}\right)\left\{\sigma_{Z Z}-v \sigma_{X X}-v \sigma_{Y Y}\right\}
\end{aligned}
$$

The assumed displacement constraint entails $\varepsilon_{Z Z}=0$. Then

$$
\sigma_{Z Z}=v \sigma_{X X}+v \sigma_{Y Y}
$$

For Case 1, let the stress on the lateral surfaces be equal: $\sigma_{X X}=\sigma_{Y Y}$. Then

$$
\varepsilon_{X X}=\left(\frac{1}{E}\right)\left\{\sigma_{X X}-v \sigma_{X X}-v^{2} \sigma_{X X}\right\}=\left(\frac{\sigma_{X X}}{E}\right)(1-2 v)(1+v) .
$$

The compliance for transverse two-dimensional bulk deformation is then

$$
\frac{\varepsilon_{X X}}{\sigma_{X X}}=\left(\frac{1}{E}\right)(1-2 v)(1+v)
$$

For stability with respect to transverse deformation, this compliance must be positive, so

$$
\text { if, } E>0, \quad v>-1 \quad \text { and } \quad v<0.5 \text {. }
$$

This is the normal range for stability of a bulk elastic solid with all surfaces free. 
The condition $\varepsilon_{X X} / \sigma_{X X}>0$ may also be satisfied by the following:

$$
E<0 \quad \text { and } \quad(1-2 v)(1+v)<0 .
$$

The range of Poisson's ratio can then be satisfied by $v<-1$, in the regime of strong ellipticity or by $v>1 / 2$ which fails strong ellipticity.

For Case 2, let the stress on one lateral surface vanish: $\sigma_{Y Y}=0$. Apply a controlled stress $\sigma_{X X}$ to the remaining two parallel surfaces. Calculating the compliance $\varepsilon_{X X} / \sigma_{X X}$ as mentioned, the following conditions are obtained for positive transverse compliance. For $E>0,|v|<1$, and for $E<0,|v|>1$. These conditions are less restrictive than those of Case 1 .

For Case 3, let the stress on the lateral surfaces be a shear stress: $\sigma_{X X}=-\sigma_{Y Y}$. Requiring the corresponding transverse compliance to be positive, the condition $G>0$ is obtained. This is one of the requirements of strong ellipticity. Since we assume that to be satisfied throughout, Case 3 imposes no additional constraint on the moduli.

For Case 4, consider stability with respect to formation of dimples on the lateral surfaces. To that end, the ratio of deformation $u$ to localized concentrated load $P$ normal to the lateral surface is given by the Boussinesq solution [7] with $r$ as the distance from the point load,

$$
\frac{u_{Z}}{P}=-\frac{(1-2 v)(1+v)}{2 \pi E r}, \quad \frac{u_{X}}{P}=\frac{\left(1-v^{2}\right)}{\pi E r} .
$$

The conditions for positive compliance, hence stability with respect to this mode of deformation are identical to those of Case 1.

In summary, a bar of material in a simple displacement-controlled tension/compression test is stable with respect to elementary transverse deformations provided that either of the two conditions is met. The first condition $E>0, v>-1$, and $v<0.5$, corresponds to the usual range of properties for stability of an unconstrained block. The second condition is $E<0$ and $v<-1$. This suggests that negative moduli could be measured in such a test; however, we make the following caveat. Demonstration of stability requires that all possible modes of instability be evaluated. In a continuous medium, there is an infinite number of such modes; study of these will be conducted in the future.

\section{COMPOSITES WITH INCLUSIONS OF NEGATIVE BULK MODULUS}

Properties of composites with granular inclusions of negative bulk modulus are calculated using the Hashin-Shtrikman 'lower' formulae, as follows [26], for shear modulus $G$ and for bulk modulus $K$ of an elastic composite,

$$
\begin{aligned}
& G_{L}=G_{2}+\frac{V_{1}}{\left(1 /\left(G_{1}-G_{2}\right)\right)+\left(6\left(K_{2}+2 G_{2}\right) V_{2}\right) /\left(5\left(3 K_{2}+4 G_{2}\right) G_{2}\right)} . \\
& K_{L}=K_{2}+\frac{\left(V_{1}\left(K_{1}-K_{2}\right)\left(3 K_{2}+4 G_{2}\right)\right)}{\left(\left(3 K_{2}+4 G_{2}\right)+3\left(K_{1}-K_{2}\right) V_{2}\right)} .
\end{aligned}
$$


Here subscript 1 indicates the inclusion and subscript 2 indicates the matrix; $V$ represents the corresponding volume fraction. Composites which attain the Hashin-Shtrikman formulae for bulk modulus have a morphology in which the composite is filled with coated spheres of different sizes but identical ratio of sphere size to coating thickness. The attainment is exact for the bulk modulus [27] and approximate for the shear modulus. Hierarchical laminates [28] exactly attain the shear modulus formula. The HashinShtrikman formulae represent bounds on the behavior of any isotropic composite, with the tacit assumption that the modulus of each phase is positive. In the present work, the moduli are allowed to be complex, giving rise to viscoelasticity. Specifically, $G^{*}=G+i G^{\prime \prime}$, in which $i=\sqrt{-1}$. The mechanical damping is $\tan \delta+\operatorname{Im}\left\{G^{*}\right\} / \operatorname{Re}\left\{G^{*}\right\}$ with $\delta_{G}$ as the phase angle between the stress and strain sinusoids. Similarly, the bulk modulus $K$ becomes a complex quantity $K^{*}$. For viscoelastic composites, the dynamic elastic-viscoelastic correspondence principle [29,30] is applied to the solutions for elastic constants of composites so that all elastic constants become complex quantities [31].

Figures 4-7 show the results of the composite analysis. As shown in Figures 4 and 5, there is no effect due to the bulk modulus $K_{1}$ of the inclusions on the composite shear modulus; however, there is a strong effect on both the composite bulk modulus and Young's modulus. Inclusions of negative bulk modulus give rise to a reduced Young's modulus, or to an extreme high value of Young's modulus, depending on the inclusion of bulk modulus value as shown in Figure 4. Also shown is a giant peak in the extensional mechanical damping $\tan \delta_{E}$, much larger than the assumed matrix damping $\tan \delta_{G}=0.02$. This behavior is similar to that of composites with inclusions of negative shear modulus [2]. The difference is that if $G<0$, the inclusions must be sufficiently small to suppress the

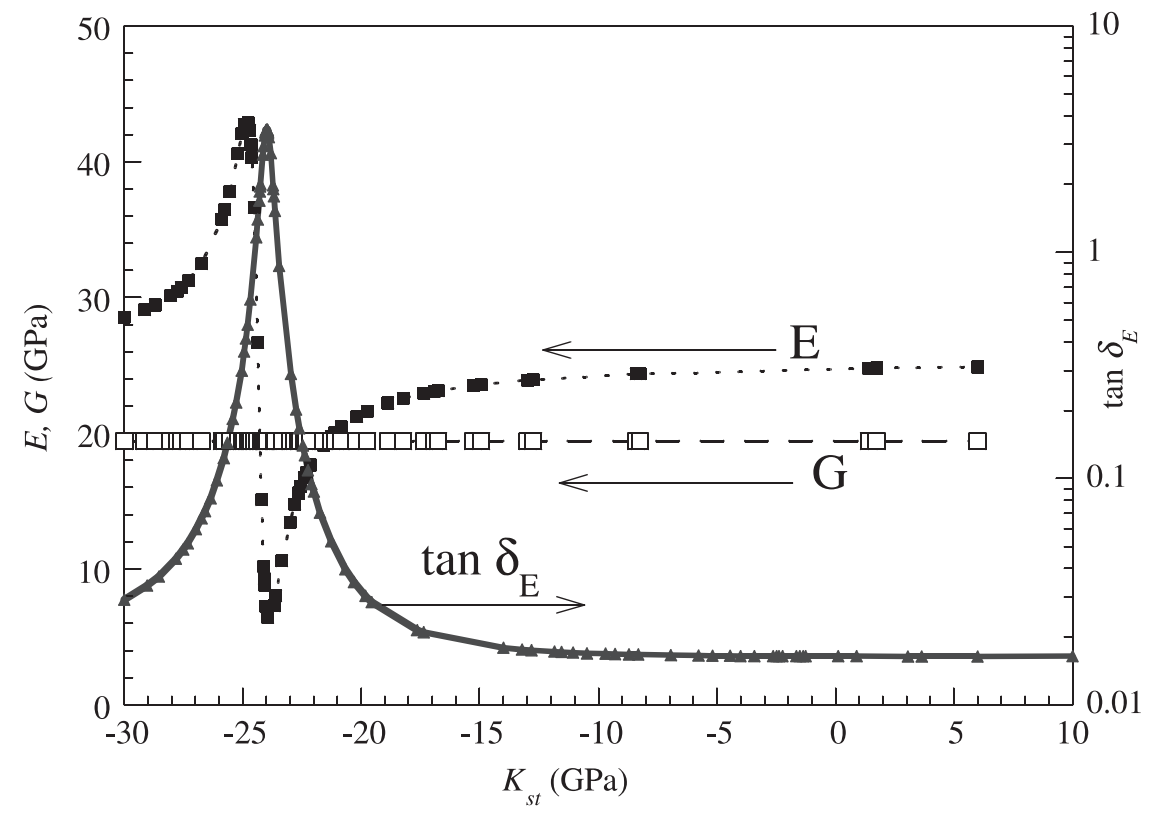

Figure 4. Composite Young's modulus $E$ (solid squares, short dash curve), mechanical damping tan $\delta_{E}$ (triangles, solid curve) and shear modulus G (open squares, long dash curve) vs inclusion bulk modulus (allowing negative values) in a dilute Hashin-Shtrikman composite with $5 \%$ by volume inclusions. Matrix properties are $G_{m}=19.2 \mathrm{GPa}, \tan \delta_{\mathrm{G}}=0.02, K_{m}=41.6 \mathrm{GPa}, v_{m}=0.3, \tan \delta_{K}=0$. 


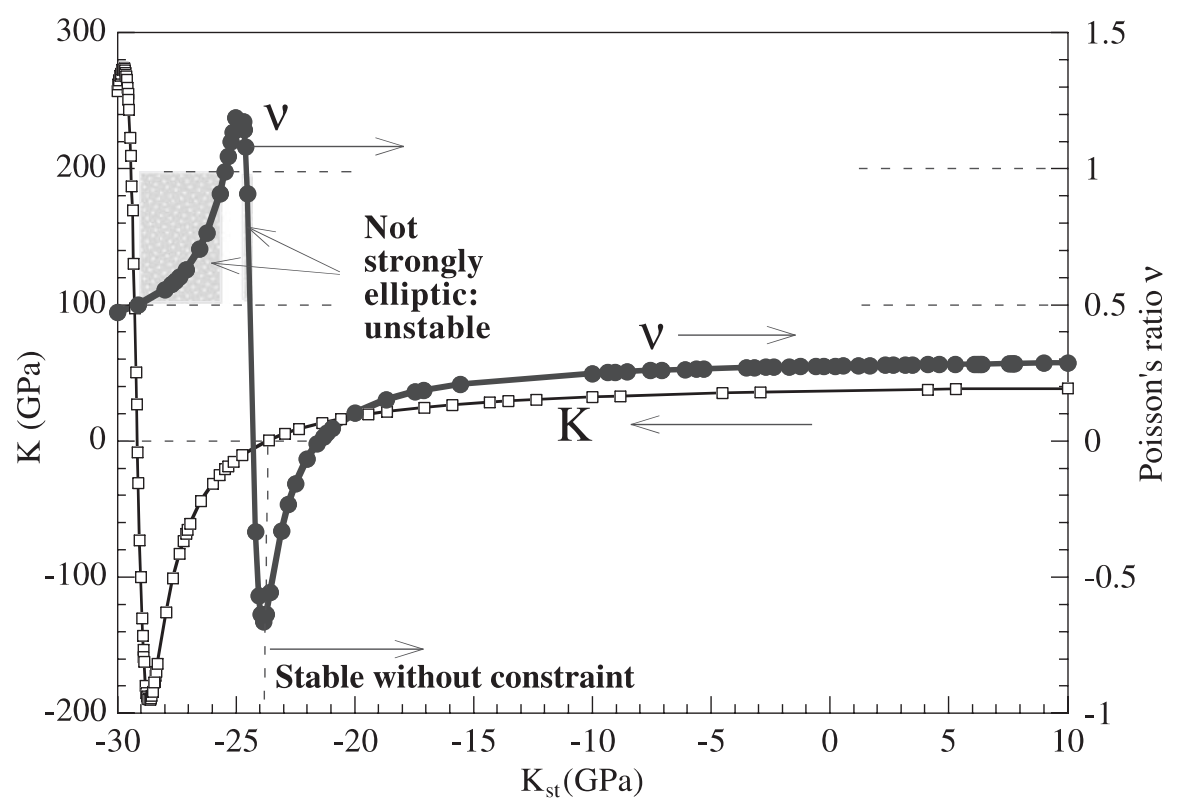

Figure 5. Composite bulk modulus $K$ (real part) (open squares, thin curve) and Poisson's ratio v (real part) (solid circles, thick curve) vs inclusion bulk modulus (allowing negative values) in a dilute Hashin-Shtrikman composite with $5 \%$ by volume inclusions. Constituent properties are as in Figure 4. Dash lines indicate values of properties ( $K=0$ and $v=0.5,1)$ which govern stability. Shaded regions denote failure of strong ellipticity, hence instability with respect to band formation.

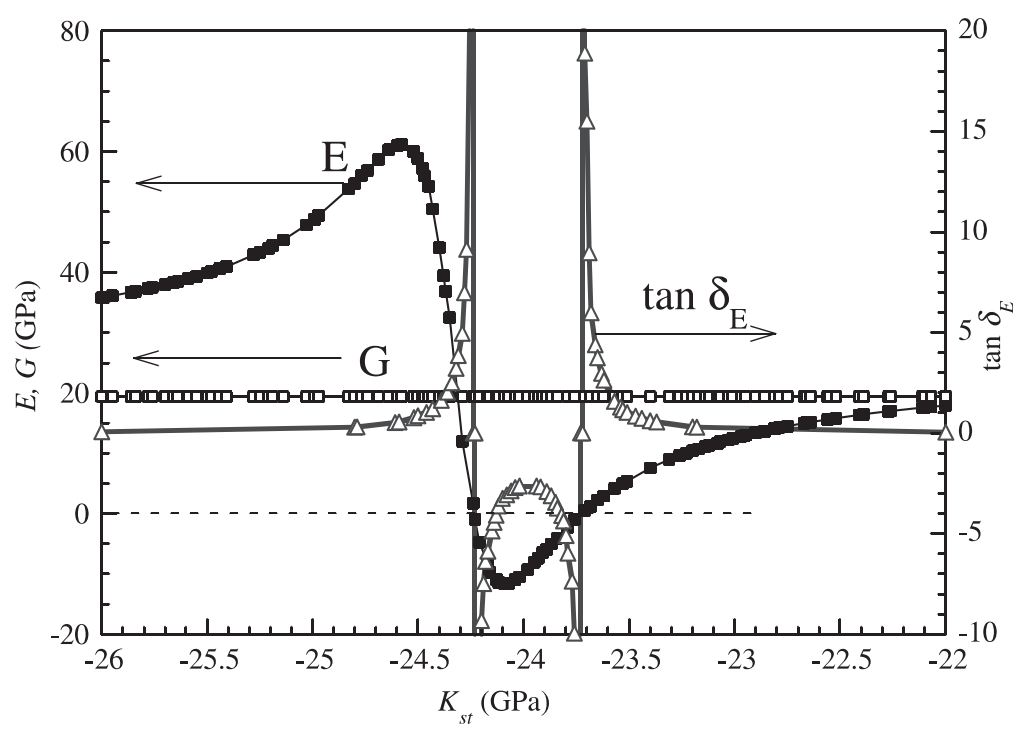

Figure 6. Detail of anomaly. Composite Young's modulus $E^{\prime}$ (real part) (solid squares), mechanical damping $\tan \delta E$ (triangles, thick curve) and shear modulus $G^{\prime}$ (real part) (open squares) vs inclusion bulk modulus (allowing negative values) in a dilute Hashin-Shtrikman composite with $5 \%$ by volume inclusions. Matrix properties are $G_{m}=19.2 \mathrm{GPa}, \tan \delta_{G}=0.01, K_{m}=41.6 \mathrm{GPa}, v_{m}=0.3, \tan \delta_{K}=0$. Dash line indicates zero modulus. 


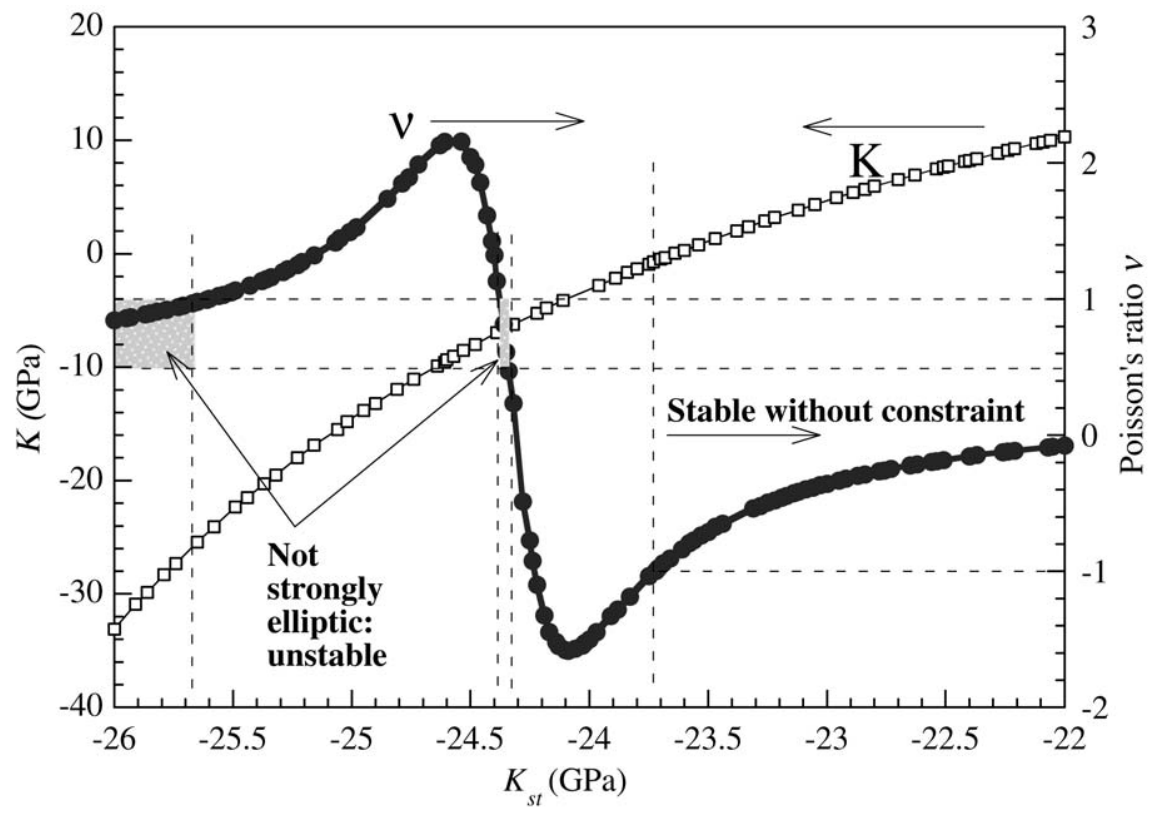

Figure 7. Details of anomaly. Composite bulk modulus K (real part) (open squares, thin curve) and Poisson's ratio v (real part) (solid circles, thick curve) vs inclusion bulk modulus. Assumed constituent properties are as in Figure 6. Dash lines indicate values of properties which govern stability of the composite as a continuum. Shaded regions denote failure of strong ellipticity, hence instability with respect to band formation.

instability associated with the domain formation. By contrast, there is no such restriction on the size of inclusions with $K<0$. For the parameters chosen, $E^{\prime}$ and $\mathrm{G}^{\prime}$ are positive throughout the range. It is also noted that $K_{L}>0$ for $K_{1}>-23.6 \mathrm{GPa}$. This region of positive composite bulk modulus encompasses part of the anomaly in $E$ and its damping (they are both positive in this example). The material in this example is strongly elliptic in portions of the transition region. Specifically, the region to the right in Figure 5 for which the Poisson's ratio is $<0.5$ includes the dip in $E$ and much of the peak in its damping, $\tan \delta_{E}$. Poisson's ratio exceeds 1 in the left shoulder of the peak in E. Again, strong ellipticity entails stability with respect to the band formation, a negative modulus implies a surface constraint is required to achieve an overall stability.

A detailed view of regions of stability is shown in Figures 6 and 7. Here, the matrix damping is reduced to $\tan \delta_{G}=0.01$. Such a small damping allows Young's modulus $E$ to go negative for certain values of inclusion stiffness. In this case damping becomes singular in the vicinity of the stability boundary. Such behavior is similar to that of a lumped system [32] with a finite number of degrees of freedom, in which a rigorous stability analysis was conducted. In the present distributed case, there are an infinite number of degrees of freedom, therefore a full analysis of the stability will be conducted in future.

Negative stiffness, as considered in the present work, is a result of elastic stored energy at equilibrium. It does not depend on resonance or other inertial effects. This is in contrast to the negative dielectric permittivity recently reported [33] and analyzed [34], as well as to the high permittivity analyzed [35] for resonant layered spheres. Negative dielectric properties are a consequence of the microstructural resonance; they occur over an extremely 
narrow range of frequency. As for composites with a negative stiffness constituent, the peaks and anomalies predicted in the present analysis and observed experimentally $[3,4]$ do not depend on resonance or inertial effects. The cancellation of terms of positive and negative stiffness in the denominator of Equations (16) and (17) gives rise to the peaks and anomalies. In resonant systems, there is a mathematically similar but physically different cancellation of terms associated with elasticity (or with dielectric permittivity) and with inertia. Sharp peaks in properties analyzed in dielectric composites with a constituent of negative permittivity [36] were considered to be of concern. Indeed, in the context of bounds, such composites may not obey some of the assumptions made in the bounding theorems. However, Equations (16) and (17) are exact solutions for the behavior of particular composite microstructures. The question of over what domain the solutions are unique and stable is a subject of current investigation.

\section{CONCLUSIONS}

Composites with spherical inclusions of negative bulk moduli exhibit anomalies in the composite bulk modulus and Young's modulus (and in the corresponding mechanical damping) but not in the shear modulus. Damping can tend to infinity in a region for which the composite is globally stable without constraint. Negative bulk modulus is shown to be possible in a prestrained lattice and in several crystalline materials.

\section{ACKNOWLEDGMENT}

The authors are grateful for a grant, CMS-0136986, from NSF. We thank W. J. Drugan and R. Carpick for discussions and encouragement.

\section{REFERENCES}

1. Thompson, J.M.T. (1979). Stability Prediction through a Succession of Folds, Phil. Trans. Royal Soc. Lond., 292: 1-23.

2. Lakes, R.S. (2001). Extreme Damping in Composite Materials with a Negative Stiffness Phase, Physical Review Letters, 86: 2897-2900.

3. Lakes, R.S. (2001). Extreme Damping in Compliant Composites with a Negative Stiffness Phase, Philosophical Magazine Letters, 81: 95-100.

4. Lakes, R.S., Lee, T., Bersie, A. and Wang, Y.C. (2001). Extreme Damping in Composite Materials with Negative Stiffness Inclusions, Nature, 410: 565-567.

5. Falk, F. (1980). Model Free Energy, Mechanics, and Thermodynamics of Shape Memory Alloys, Acta Metall., 28: 1773-1780.

6. Salje, E. (1990). Phase Transitions in Ferroelastic and Co-Elastic Crystals, Cambridge University Press, Cambridge, UK.

7. Timoshenko, S.P. and Goodier, J.N. (1970). Theory of Elasticity, 3rd edn, McGraw-Hill, New York, NY.

8. Lakes, R.S. (1987). Foam Structures with a Negative Poisson's Ratio, Science, 235: 1038-1040.

9. Lakes, R.S. (1993). Advances in Negative Poisson's Ratio Materials, Advanced Materials, 5: 293-296.

10. Milton, G. (1992). Composite Materials with Poisson's Ratios Close to -1, J. Mech. Phys. Solids, 40: 1105-1137. 
11. Knowles, J.K. and Sternberg, E. (1978). On the Failure of Ellipticity and the Emergence of Discontinuous Gradients in Plane Finite Elastostatics, J. Elasticity, 8: 329-379.

12. Bramble, J.H. and Payne, L.E. (1963). On the Uniqueness Problem in the Second Boundary Value Problem in Elasticity, In: Proc. Fourth National Congress of Applied Mechanics, pp. 469-473.

13. Sokolnikoff, I.S. (1983). Mathematical Theory of Elasticity, Krieger, Malabar, FL.

14. Bazant, Z. and Cedolin, L. (1991). Stability of Structures, Oxford University Press, Oxford.

15. Rosakis, P., Ruina, A. and Lakes, R.S. (1993). Microbuckling Instability in Elastomeric Cellular Solids, J. Materials Science, 28: 4667-4672.

16. Lakes, R.S. and Drugan, W.J. (2002). Dramatically Stiffer Elastic Composite Materials due to a Negative Stiffness Phase?, J. Mechanics and Physics of Solids, 50: 979-1009.

17. Berglund, K. (1977). Investigation of a Two-Dimensional Model of a Micropolar Continuum, Archives of Mechanics, 29: 383-392.

18. Kindler, B., Finsterbusch, D., Graf, R., Ritter, F., Assmus, W. and Lüthi, B. (1994). Mixedvalence Transition in $\mathrm{YbInCu}_{4}$, Phys. Rev. B, 50: 704-707.

19. Lawrence, J.M., Riseborough, P.S. and Parks, R.D. (1981). Valence Fluctuation Phenomena, Rep. Prog. Phys., 44: 1-84.

20. Francheschi, E. and Olcese, G.L. (1969). A New Allotropic Form of Cerium due to its Transition under Pressure to the Tetravalent State, Phys. Rev. Lett., 22: 1229-1300.

21. Bustingorry, S., Jagla, E.A. and Lorenzana, J. (2003). Solid-solid Volume Collapse Transitions are Zeroth Order, arXiv:comd-mat/0307134, Vol. 1, 7 Jul.

22. Young, D.A. (1991). Phase Diagrams of the Elements, p. 106, University of California Press, Berkeley, CA.

23. Hedges, E.S. and Higgs, J.Y. (1952). Preparation of Grey Tin, Nature, 169: 621-622.

24. Bergman, D.J. and Halperin, B.I. (1976). Critical Behavior of an Ising Model on a Cubic Compressible Lattice, Phys. Rev. B, 13: 2145-2175.

25. Lakes, R.S. (1991). Deformation Mechanisms of Negative Poisson's Ratio Materials: Structural Aspects, J. Materials Science, 26: 2287-2292.

26. Hashin, Z. and Shtrikman, S. (1963). A Variational Approach to the Theory of the Elastic Behavior of Multiphase Materials, J. Mech. Phys. Solids, 11: 127-140.

27. Hashin, Z. (1962). The Elastic Moduli of Heterogeneous Materials, J. Applied Mechanics, 29: $143-150$.

28. Milton, G.W. (1986). Modelling the Properties of Composites by Laminates, In: Erickson, J.L., Kinderlehrer, D., Kohn, R. and Lions, J.L. (eds), Homogenization and Effective Moduli of Materials and Media, pp. 150-175, Springer Verlag, Berlin.

29. Hashin, Z. (1965). Viscoelastic Behavior of Heterogeneous Media, J. Appl. Mech., Trans. ASME, 32E: 630-636.

30. Read, W.T. (1950). Stress Analysis for Compressible Viscoelastic Materials, J. Appl. Phys., 21: 671-674.

31. Christensen, R.M. (1969). Viscoelastic Properties of Heterogeneous Media, J. Mech. Phys. Solids, 17: 23-41.

32. Wang, Y.C. and Lakes, R.S. (2005). Stability of Negative Stiffness Viscoelastic Systems, Quarterly of Applied Math., 63: 34-55.

33. Shelby, R.A., Smith, D.R. and Schultz, S. (2001). Experimental Verification of a Negative Index of Refraction, Science, 292: 77-79.

34. Smith, D.R. and Kroll, N. (2000). Negative Refractive Index in Left-handed Materials, Phys. Rev. Lett., 85: 2933-2936

35. Nicorovici, N.A., Mcphedran, R.C. and Milton, G.W. (1994). Optical and Dielectric Properties of Partially Resonant Systems, Phys. Rev. B, 49: 8479-8482.

36. Mackay, T.G. and Lakhtakia, A. (2004). A Limitation of the Bruggeman Formalism for Homogenization, Optics Communications, 234: 35-42. 